\title{
Photochemical and photobiological properties of furocoumarins and homologues drugs
}

\author{
F. Bordin \\ Department of Pharmaceutical Sciences of Padova University, Padova, Italy
}

\begin{abstract}
Furocoumarins are natural photosensitizing drugs used in PUVA photochemotherapy and in photopheresis. Their therapeutic effectiveness is connected to the lesions they induce to various cell components, membranes, ribosomes, mitochondria, and in particular to DNA, damaged by formation of monofunctional adducts and of inter-strand cross-links (ISC). ISC represent a severe damage, mainly correlated to the main side effects observed in photochemotherapy, skin phototoxicity and genotoxicity. Searching for new monofunctional derivatives, two tetramethylfuroquinolinones, 1,4,6,8-tetramethyl-2H-furo[2,3-h]quinolin-2one (FQ) and 4,6,8,9-tetramethyl-2H-furo[2,3-h]-quinolin-2-one (HFQ) were studied. Both compounds are very active; however while FQ produced many chromosomal aberrations and strong skin erythemas, HFQ practically did not induce such side effects. FQ and HFQ formed high levels of monoadducts but no ISC in DNA, but both provoked many DNA-protein cross-links (DPC). FQ induced these lesions by a biphotonic reaction: at first a furan-side monoadduct is formed, which is then converted into a DPC; thus the FQ molecule seemed to form the bridge between DNA and proteins. HFQ formed DPC by a single step (DPC at zero length, like UVC). For these features, HFQ appears to be the first molecule belonging to a new class of active but not phototoxic drugs for photomedicine.
\end{abstract}

\section{INTRODUCTION}

Furocoumarins are active photosensitizing drugs widely used in photomedicine [1], in research on the structure of various biological macromolecules [2] and on DNA repair [3]. Some of them are natural compounds present in several plants, such as Psoralea corylifolia, Amni Majus and Angelica Archangelica (see Figure 1 for the molecular structure of some natural derivatives). Furocoumarins are used in photomedicine<smiles>O=c1ccc2cc3ccoc3cc2o1</smiles>

Psoralen<smiles>COc1c2ccoc2cc2oc(=O)ccc12</smiles>

5-Methoxypsoralen (5-MOP, Bergapten)

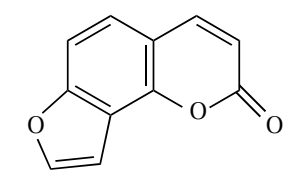

Angelicin (Isopsoralen)<smiles>COc1c2occc2cc2ccc(=O)oc12</smiles>

8-Methoxypsoralen (8-MOP, Xanthotoxin)
Figure 1. Molecular structure of some natural occurring furocoumarins.

from centuries: in fact, ancient Egyptian and Indian physicians used extracts of leaves, seeds or roots from plants containing furocoumarins for the cure of vitiligo since thousands years BC [4]. These preparations were applied on the skin or ingested and the patient was then exposed to sunlight. In seventy years, photochemotherapy PUVA (psoralen + UVA) has been introduced: 8-methoxypsoralen (8-MOP), the active principle presented in Amni Majus, was administered orally; two hours later the skin area to be cured was exposed to UVA light (320-400 nm) [5]. PUVA is effective against various skin diseases, such as vitiligo, psoriasis, alopecia aerata, atopic dermatitis, mycosis fungoides, etc. [1]. More recently the extracorporeal photochemotherapy or photopheresis has been introduced [6]. According to this therapy 8-MOP is administered orally; after two hours about 0.5 litres of blood were taken out by the patient, the lymphocytes were isolated and exposed to UVA light. Then, lymphocytes were returned back to the patient. This treatment is practically an auto vaccination against the same lymphocytes; because they are specific for the disease, a selective immuno modulation is induced, without dangers of opportunistic infections. FDA approved photopheresis for the cure of T-cell lymphoma but it is effective against various autoimmune diseases and in the prevention of rejection in organ transplantation [7].

By UVA irradiation furocoumarins induce various lesions in a living cell: damage to unsatured fatty acids and lecithins of membranes [8,9] and to ribosomes $[10,11]$. However the main damage is provoked in DNA [12]. Linear furocoumarins (psoralens) have two reactive sites in their molecule, the double bonds at 3,4 positions at pyron ring and at $4^{\prime}, 5^{\prime}$ at furan one. In the dark furocoumarins intercalate into base pairs of DNA without significant consequences; however, by light activation they react with pyrimidine bases via a $C_{4}$ cycloaddition, forming covalent monoadducts (MA) engaging 3,4 (pyron side) or $4^{\prime}, 5^{\prime}$ (furan side) positions. Furan side monoadducts can absorb the UVA light and react further with a second pyridine bases, thus forming a covalent bridge between the two DNA strands (inter-strand cross-links, ISC) [13]. The formation of such bifunctional adducts represents a severe damage, 
which is regarded as mainly responsible for the side effects of PUVA therapy, the induction of skin erythemas [1] and for genotoxicity [14]. Thus, with the aim to obtain furocoumarins having a reduced toxicity, various authors prepared and studied several monofunctional derivatives, incapable of forming ISC. A monofunctional furocoumarin can be obtained by several ways: blocking one of its two photoreactive sites by insertion of a suitable group, as for 3-carbethoxypsoralen [15], or by cumulating a fourth nucleus, e.g., the pyridopsoralens having a pyridine condensed at 3,4 positions [16] and benzopsoralens in which the fourth nucleus is a benzenic one at $4^{\prime}, 5^{\prime}$ position [17]. We also studied several new angelicin derivatives, which, for the geometric parameters of their angular molecular structure, can hardly induce ISC [18]. Thus, we obtained various active and interesting angelicins; the best one is certainly 4,6,4'-trimethylangelicin [18]. Recently we also studied some homologues drugs which can be considered angelicin isosters; the most interesting compounds are two angular furoquinolinones, FQ $(1,4,6,8$ tetramethyl-2H-furo[2,3-h]quinolin-2-one, according to JUPAC) [19] and HFQ (4,6,8,9-tetramethyl-2H-furo[2,3$\mathrm{h}$-quinolin-2-one) having a nitrogen atom replacing the oxygen at 1 position; the main difference between these two derivatives is the presence or the absence of a methyl group at the nitrogen atom. Figure 2 shows their molecular structure.<smiles>Cc1cc2c(o1)c(C)cc1c(C)cc(=O)n(C)c12</smiles>

FQ<smiles></smiles>

HFQ
Figure 2. Molecular structure of the tetramethylfuroquinolinones.

\section{MAIN PROPERTIES OF FUROQUINOLINONES}

Both FQ and HFQ are characterized by a strong photosensitizing activity. In fact, upon UVA irradiation, both compounds induce a dramatic killing effect in $\mathrm{CHO}$ cells, even when they were employed in very mild experimental conditions. As we can see in Figure 3, there are not significant differences between the survival curves generated by FQ and HFQ. On the contrary, 8-MOP, tested at a double concentration, induced a very small effect. However, studying their capacity of inducing erythemas on the skin, we obtained a surprising result: while FQ appeared to be as phototoxic as 8-MOP, HFQ was nearly inactive. The threshold doses for erythema induction, that is the minimum amount of drug and of UVA light necessary to induce a barely visible erythema on albino guinea-pig skin, are shown in Table 1. FQ is only slightly more active than 8-MOP, while HFQ is poorly effective.

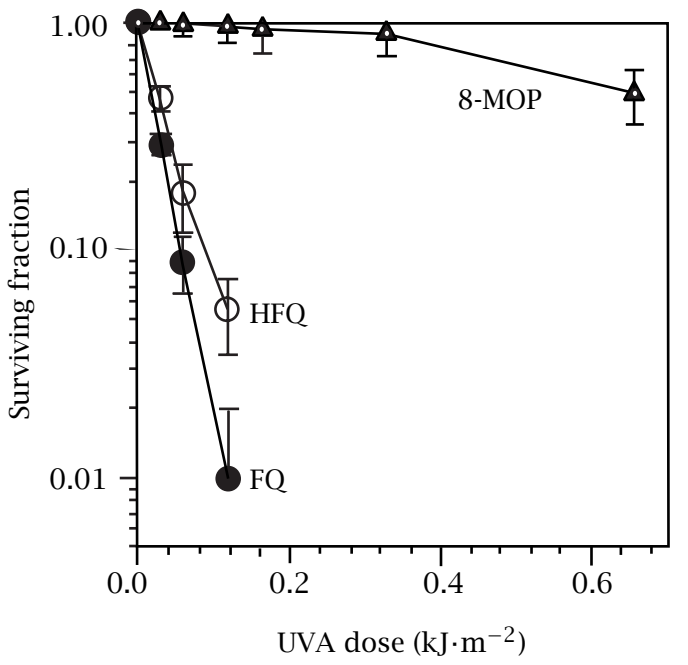

Figure 3. Clonal growth of CHO: cells were exposed to UVA light in the presence of the drug (FQ and HFQ 2.3 $\mu \mathrm{M}$; 8-MOP $4.6 \mu \mathrm{M})$ and then the capacity of cells of forming clones was assayed.

Table 1. Threshold doses for erythemas induction on guinea pig skin.

Compounds were applied on the skin as a $0.1 \%$ methanolic solution and the skin was exposed to UVA light. The animals were then kept in the dark and observed for 3 days. The dose unit is a parameter proportional to the activity, defined as follows: $1 /\left(\left(\mu \mathrm{M} \cdot \mathrm{cm}^{-2}\right)\left(\mathrm{kJ} \cdot \mathrm{m}^{-2}\right)\right)$ in which $\mu \mathrm{M} \cdot \mathrm{cm}^{-2}$ is the furocoumarin amount applied to $\mathrm{a} \mathrm{cm}^{2}$ of the skin and $\mathrm{kJ} \cdot \mathrm{m}^{-2}$ is the UVA dose.

\begin{tabular}{lcccc}
\hline Compound & $\mu \mathrm{M} \cdot \mathrm{cm}^{-2}$ & $\mathrm{~kJ} \cdot \mathrm{m}^{-2}$ & $\begin{array}{c}\text { Dose } \\
\text { unit } \times 10^{-2}\end{array}$ & $\begin{array}{c}\text { Relative } \\
\text { activity }\end{array}$ \\
\hline 8-MOP & 4.6 & 5 & 4.3 & 1 \\
FQ & 3.3 & 5 & 6 & 1.39 \\
HFQ & 20 & 25 & 0.2 & 0.04 \\
\hline
\end{tabular}

The skin phototoxicity is the furocoumarin photosensitising effect known from the longest time; in fact, in the past, a non-skin phototoxic compound was regarded as totally inactive. At present we don't know what is the mechanism of erythema formation, even if several hypothesis have been suggested: the generation of active species of oxygen, such as singlet oxygen [20] or the ISC induction [1]. However until now, all experimental data bore out neither of these hypotheses. Nevertheless, in 1990, Ortel et al., using the socalled double irradiation protocol, observed that the erythemas on human skin generated by 8-MOP are induced by a two steps reaction, as happen for ISC formation [21]. It is well known that furocoumarins induce ISC by the sequential absorption of two photons; the first one yields to the formation of a furan side monoadduct and the second photon converts it into an inter-strand cross-link. The double-irradiation is an important method which allows a selectively study of the consequence of MA and ISC [19]. After a small UVA dose, which provokes mainly the formation of 
monoadducts, the unbound furocoumarin molecules are removed by washing and the biological system is exposed again to the light. During the second irradiation step some monoadducts can be converted into ISC without an increase of the total number of the lesions.

\section{FUROCOUMARINS INDUCE DNA-PROTEIN CROSS-LINKS}

On the bases of these data, we suggested that furocoumarins could undergo to another kind of biphotonic reaction; because they can link covalently both to DNA and to proteins [22], we supposed they can form DNAprotein cross-links (DPC). Since it is impossible to detect such lesions in simplified systems, we studied them in whole mammalian cells, using alkaline elution [19]. Thus, we observed that linear furocoumarins induce DPC, while angular ones, like angelicin derivatives, are less effective [23]. Therefore, the study of the mechanism and of the consequence of DPC appeared to be very difficult, because linear furocoumarins form both DPC and ISC.

The solution of this problem was found with FQ and $\mathrm{HFQ}$, the two furoquinolinones above mentioned. Actually, as reported in Table 2 and contrary to 8-MOP, both compounds are incapable of inducing ISC, while they can form DPC to noticeable extents.

Table 2. Damage induced in DNA $(8-M O P=1)$. The data are expressed as relative activity in comparison to 8-MOP. (a) detected in $\mathrm{CHO}$ cells by alkaline elution; (b) estimated in vitro in the photoreaction with calf thymus DNA.

\begin{tabular}{cccc}
\hline Compound & ISC $^{(\mathrm{a})}$ & DNA binding & \\
\hline HFQ & 0.01 & 5.70 & 2.40 \\
FQ & 0.01 & 9.56 & 8.50 \\
8-MOP & 1.0 & 1.0 & 1.0 \\
\hline
\end{tabular}

\section{MECHANISMS OF DPC FORMATION}

Therefore, we studied the formation of DPC and some their biological consequences using FQ and HFQ. At first, we studied DPC formation in CHO cells using alkaline elution and the above mentioned doubleirradiation protocol [19]. Figure 4 shows the results thus obtained. Furan-side monoadducts induced by FQ can produce a number of DPC, with a gradual increase of their amount according to the UVA dose delivered in the second step. On the contrary, with HFQ, after the second step the DPC number remained practically unchanged.

Therefore, it is evident that FQ induces a number of DPC by a two step mechanism, while HFQ can form this lesion only by a one step reaction. Consequently, we can suppose that FQ induces a new diadduct in which its molecule forms the bridge between DNA and protein (DPC at length greater than zero); on the contrary, HFQ induces DPC probably by energy transfer (DPC at zero length).

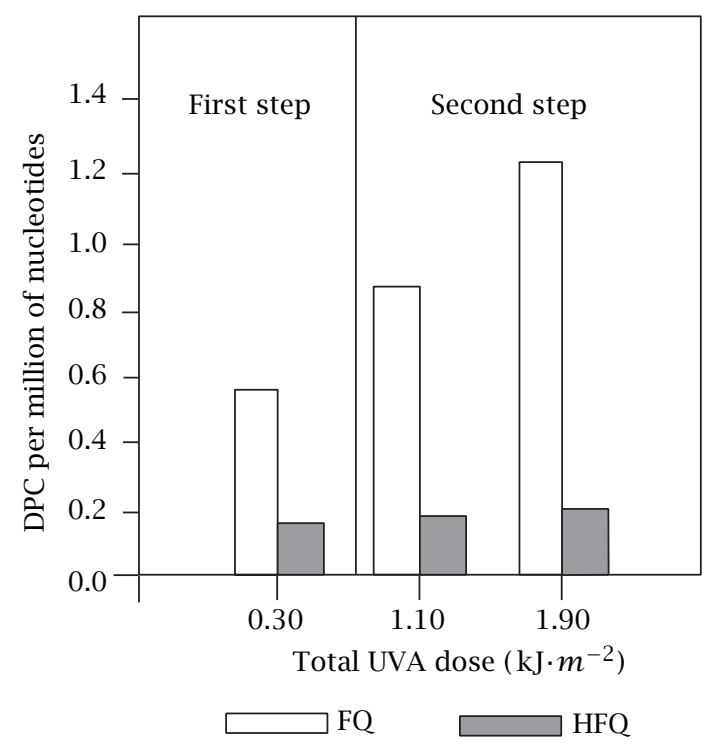

Figure 4. DPC formation in $\mathrm{CHO}$ cells by the doubleirradiation method: $\mathrm{CHO}$ cells were exposed at a first irradiation step $\left(0.06 \mathrm{KJ} \cdot \mathrm{m}^{-2}\right)$ in the presence of $2.3 \mu \mathrm{M} \mathrm{FQ}$ or HFQ. The cells were washed and then submitted to the second step, carried out with two different UVA dose. DPC were detected by alkaline elution [25].

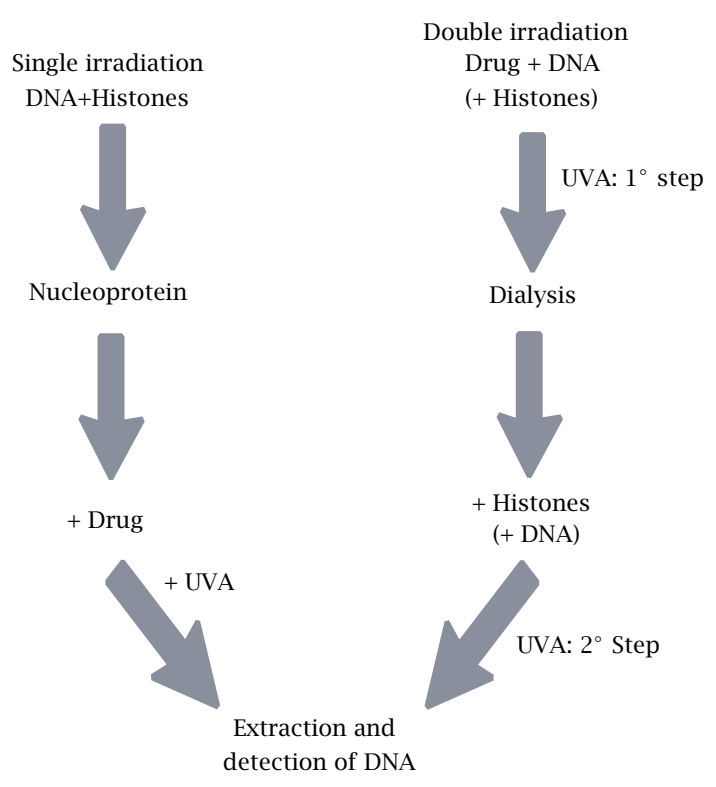

Figure 5. Scheme of the methods used for DPC detection in vitro. (1) Single irradiation: aqueous solutions of DNA, histones and the drug $(20 \mu \mathrm{M})$ were mixed together, and the mixture was exposed to UVA light $\left(15 \mathrm{~kJ} \cdot \mathrm{m}^{-2}\right) ; D N A$ was extracted and determined by spectrophotometric determination. (2) Double irradiation: an aqueous solution of calf thymus DNA was exposed to $10 \mathrm{~kJ} \cdot \mathrm{m}^{-2}$ in the presence of the drug $(20 \mu \mathrm{M})$ and then submitted to dialysis overnight against PBS; histones from calf thymus were added to the solution which was then irradiated again $\left(15 \mathrm{~kJ} \cdot \mathrm{m}^{-2}\right)$; DNA was extracted and its amount determined as above. To check the adduct formation with proteins, histones were submitted to the first irradiation step: in this case after dialysis, DNA was added and the solution was irradiated for the second time and processed as above. 
To confirm these data we carried out some experiments in vitro; Figure 5 shows the protocols used, by single and double irradiation. In the single irradiation method, DNA and histones from calf thymus were mixed together thus obtaining a nucleoprotein complex, which was exposed, to UVA light in the presence of furocoumarins. Then DNA was extracted and its amount determined. Because the formation of DPC reduced the amount of the extractable DNA, we can have an evaluation of their amount. This protocol was also modified according to the double-irradiation method: actually, we can submit to the first step DNA or histones. After the first irradiation the free furocoumarin molecules were removed by dialysis, and the second macromolecule, histones or DNA respectively, was added to the mixture. Then it was submitted to the second UVA exposure followed by DNA extraction. Figure 6 shows the results obtained with furoquinolinones and 8-MOP using these procedures. These data are consistent with those obtained in vivo; actually, the different mechanisms of DPC formation by FQ and HFQ were confirmed by these experiments. In fact, with FQ (and 8-MOP too) using the double-irradiation protocol and treating DNA in the first step we obtained DPC to an extent very close to that achieved by irradiating the pre-formed nucleoprotein complex. On the contrary, HFQ always yielded very poor amounts of DPC by the two steps protocol. Moreover, using histones in the first step we obtained completely negative results. This means that the biphotonic reaction involving FQ (or 8MOP) requires the formation of MA during the first step.

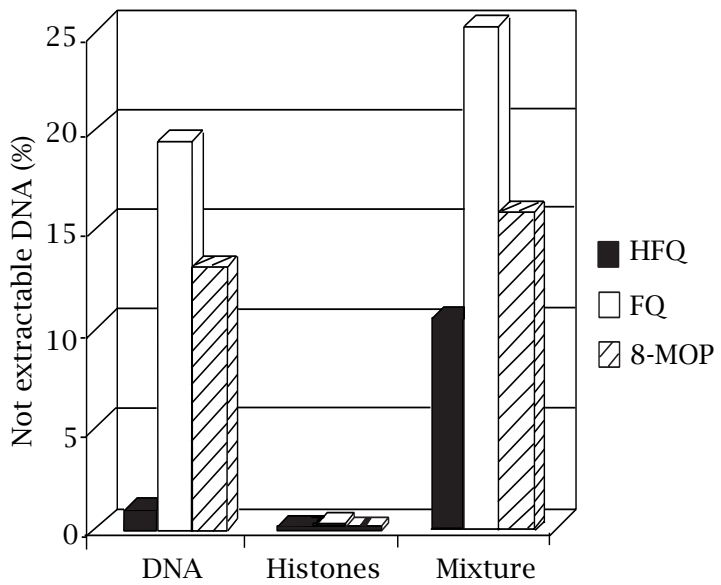

Figure 6. (1) DNA: double-irradiation procedure in which DNA was submitted to the first irradiation step. (2) Histones: double-irradiation procedure in which histones were submitted to the first irradiation step. (3) Mixture: according to single irradiation procedure DNA, histones and the drug were submitted together to a single UVA light exposure.

The behaviour of HFQ can be explained by the low absorption of its furan side monoadducts at $360 \mathrm{~nm}$, that is the maximum of emission of the lamp used in our experiments [24].

\section{BIOLOGICAL CONSEQUENCES OF DPC FORMATION}

As a first approach, we detected the formation of double-strand breaks (DSB; DNA fragmentation) by neutral elution [25]; $\mathrm{CHO}$ cells were submitted to sensitization with increasing UVA doses and then they were incubated for 24 hours before testing DNA damage. Figure 7 shows the results thus obtained. FQ induced a very large DNA fragmentation even at very low UVA doses, much more pronounced than 8-MOP; on the contrary, HFQ appeared to be ineffective. We must realize that DNA fragmentation is not provoked by the photochemical reaction, because it can be observed only after a suitable incubation time, performed after the treatment, at least 12 hours (data not shown). This means it is due to an enzymatic DNA processing that takes place during incubation, very likely by DNA repair.

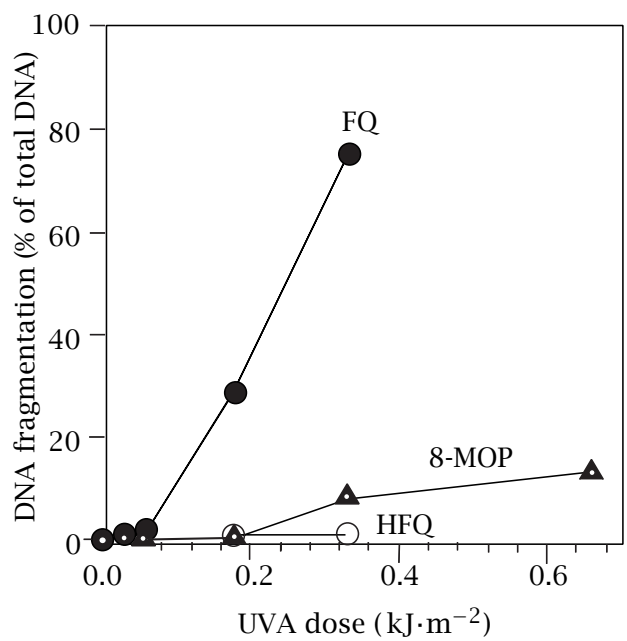

Figure 7. DSB formation by sensitisation with FQ HFQ $(2.3 \mu \mathrm{M})$ and 8 -MOP $(4.6 \mu \mathrm{M})$. CHO cells were exposed to increasing UVA doses in the presence of the drug and then incubated for 24 hours. DSB were detected by neutral elution [25].

This behaviour is consistent with the data obtained studying the capacity of furoquinolinones of forming chromosomal aberrations (see Table 3). Actually FQ induced a lot of aberrations, while HFQ gave values very close to the controls.

To have direct information of the consequences induced by DPC at length greater than zero formed by $\mathrm{FQ}$, we carried out some experiments with the doubleirradiation protocol. Because both FQ and HFQ cannot form ISC at all, we can only detect the biological effect of the formation of DPC. Figure 8 shows the data obtained with this procedure in $\mathrm{CHO}$ cells testing the formation of chromosomal aberrations. We can see that their number increases after the second irradiation step; because FQ with this mechanism induces only DPC, but not ISC, this result tells us that DPC are mainly responsible of FQ genotoxicity. On the contrary, HFQ was practically inactive. To establish this assumption, we plotted the number of aberrations scored with FQ against DPC amounts 
Table 3. Chromosomal aberrations in $\mathrm{CHO}$ cells. After treatment, CHO cells were incubated for 24 hours at $37^{\circ}$ in growth medium, and then the aberration frequency was determined.

\begin{tabular}{|l|c|c|c|}
\hline Compound & $\begin{array}{c}\text { Drug } \\
\text { concentration } \\
(\mu \mathrm{M})\end{array}$ & $\begin{array}{c}\text { UVA dose } \\
\left(\mathrm{kJ} \cdot \mathrm{m}^{-2}\right)\end{array}$ & $\begin{array}{c}\text { Total } \\
\text { aberration } \\
\text { frequency (\%) }\end{array}$ \\
\hline None & 0 & 2 & 0.8 \\
(controls) & & & \\
8-MOP & 4.6 & 1.65 & 32.5 \\
FQ & 2.3 & 0.12 & 75.6 \\
HFQ & 2.3 & 0.12 & 2.65 \\
\hline
\end{tabular}

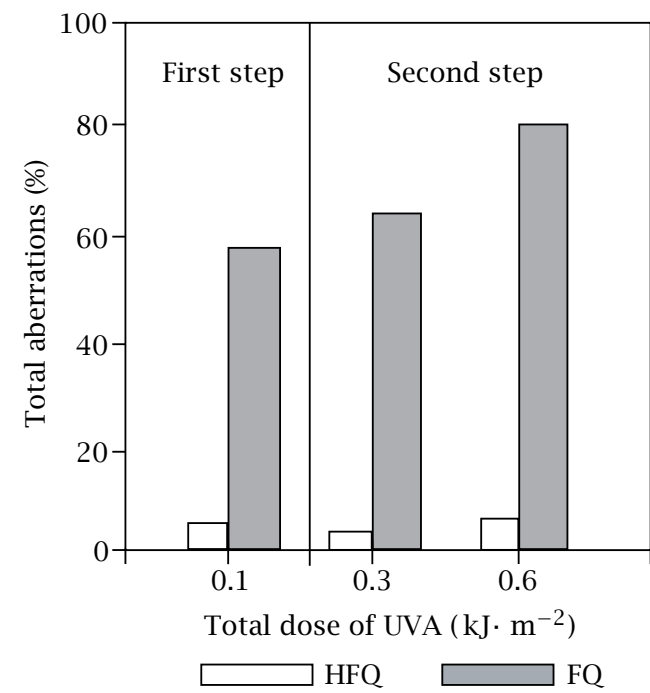

Figure 8. Chromosomal aberrations induced by the doubleirradiation protocol. The first step was carried out using a $2.3 \mu \mathrm{M}$ concentration. After several washing, CHO cells were irradiated further with different UVA doses. The aberration frequency was determined after 24 hours of incubation.

formed in the same experimental conditions. A straight line was obtained, with a good linear relationship (data not shown).

Using the same protocol, we also detected the lethality of DPC greater than zero of FQ in CHO cells; as expected, with HFQ the surviving fraction obtained after the first irradiation was not further decreased significantly by the second step. As expected, during the second irradiation step DPC formed by FQ strongly reduced the survival, according to the UVA dose, with an evident killing effect (see Figure 9).

\section{DISCUSSION}

FQ and HFQ induce the same kinds of damages into DNA, roughly to the same order of magnitude (mainly monofunctional adducts and DPC, but no ISC); in comparison with 8-MOP both compounds are much more effective. This behaviour is consistent with the very high antiproliferative activity tested on the clonal growth of

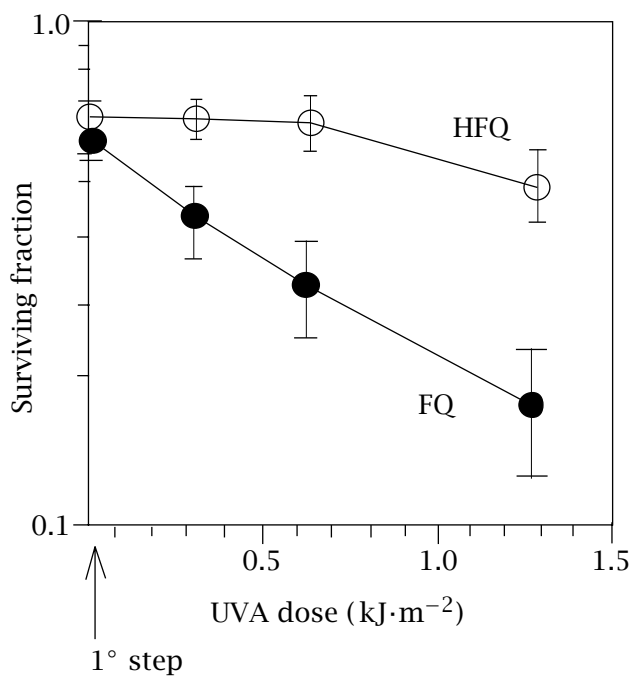

Figure 9. Clonal growth studied by the double-irradiation protocol: $\mathrm{CHO}$ cells were exposed to the first irradiation step $\left(0.06 \mathrm{KJ} \cdot \mathrm{m}^{-2}\right)$ in the presence of $2.3 \mu \mathrm{MFQ}$ or HFQ. The cells were washed and submitted to the second irradiation step. Clonal growth was then determined.

CHO cells; in this test, 8-MOP, despite its capacity of forming ISC, appeared to be poorly active, even if assayed in more severe conditions. Using the doubleirradiation method we observed that FQ could induce a number of DPC by a two steps mechanism; on the contrary, HFQ can form this lesion only by a single step one. Because both drugs cannot form ISC, we studied the antiproliferative activity of DPC using the doubleirradiation method; as expected, the second irradiation step was ineffective with HFQ, but, on the contrary, FQ induced a strong reduction of the survival.

It is well known that the formation of ISC by furocoumarins requires the sequential absorption of two photons. We found that FQ induced DPC by a similar process. Conversely, DPC formation by HFQ occurs certainly with the absorption of a single photon; therefore they are certainly cross-links at zero length, like to that formed by UVC. In conclusion, we could suppose DPC formed by the two drugs might be different. At present this is only a working hypothesis, but it seems to be consistent with the data we obtained studying the repair and the genotoxicity of such lesions. In fact, following the formation of DSB in DNA by incubating in growth medium the sensitized CHO cells, we observed with FQ a very high DNA fragmentation; with HFQ practically no DSB were formed. DSB are clearly not induced by a photochemical reaction but by enzymatic processes occurring during cell incubation after the treatment. 8-MOP, used as a reference, formed significant amounts of DSB even if to a much lower extent in comparison with FQ. We obtained the same picture studying the formation of chromosomal aberrations. FQ yielded a lot of aberrations and their number increased with the second irradiation step: this probably means they are produced by DPC induction. Even in this test, HFQ appeared to be 
practically inactive.

Checking the classic effect induced by furocoumarins, i.e., the formation of skin erythemas, we found again the same situation: FQ is almost as active as 8-MOP, while HFQ only provoked weak erythemas in more severe experimental conditions.

Therefore, DPC formed by the furoquinolinones appear to be very different, at least on the bases of their dissimilar mechanism of formation and of their biological consequences. As stated before, certainly DPC formed by HFQ are at zero length. DPC induction by FQ represents a more complicated process. Actually, even if these DPC are formed by a double-step mechanism like ISC, thus suggesting they are cross-links at length greater than zero, at present we have no direct evidences that the FQ molecule is really a physical part of the cross-links between the two macromolecules. However this seems to be much more than an interesting hypothesis, because it is supported also by some data already published [25].

Finally, HFQ seems to be an interesting drug for photomedicine. In fact, the data regarding its activity on mammalian cells together with its very low skin phototoxicity and genotoxicity suggest that it could be a new promising agent for photochemotherapy.

\section{REFERENCES}

[1] J. A. Parrish, R. S. Stern, M. A. Pathak, and T. B. Fitzpatrick, The Science of Photomedecine, p. 595, plenum Press, New York, 1982, J. D. Regan and J. A. Parrish (Eds.).

[2] G. Cimino, H. Gamper, S. Isaacs, and J. Hearst, Ann. Rev. Biochem. 54 (1985), 1151.

[3] B. van Houten, Microbiol. Rev. 54 (1990), 18.

[4] M. A. Pathak, D. M. Kramer, and T. B. Fitzpatrick, Sunlight and Man, p. 335, University Tokyo Press, Tokyo, 1974, M. A. Pathak, L. C. Harber, M. Seiji and A. Kukita (Eds.).

[5] J. A. Parrish, T. B. Fitzpatrick, L. Tanenbaum, and M. A. Pathak, New Eng. J Med. 291 (1974), 1207.

[6] R. L. Edelson, C. L. Berger, F. P. Gasparro, et al., N. Engl. J. Med. 316 (1987), 297.

[7] F. P. Gasparro, Photochem. Photobiol 63 (1996), 553.
[8] Z. Zarebska, E. Waskkowska, S. Caffieri, and F. Dall'Acqua, J. Photochem. Photobiol. B: Biol. 45 (1998), 122.

[9] Z. Zarebska, J. Photochem. Photobiol. B: Biol. 23 (1994), 101.

[10] H. Singh and J. A. Vadasz, Photochem. Photobiol. 28 (1978), 539.

[11] F. Baccichetti, C. Marzano, F. Carlassare, A. Guiotto, and F. Bordin, J. Photochem. Photobiol. B: Biol. 40 (1997), 299.

[12] E. Ben-Hur and P. S. Song, Adv. Rad. Biol. 11 (1984), 131.

[13] G. J. Hook, J. A. Heddle, and R. R. Marshall, Cytogenet. Cell Genet. 35 (1983), 100.

[14] P. Queval and E. Bisagni, Eur. J. Med. Chem. 3 (1974), 355.

[15] L. Dubertret, D. Averbeck, E. Bisagni, J. Moron, E. Moustacchi, C. Billardon, D. Papadopoulo, S. Nocentini, P. Vigny, J. Blais, R. V. Bensasson, J. C. Ronfard-Haret, E. J. Land, F. Zajdela, and C. Latarjet, Biochimie 67 (1985), 417.

[16] F. Bordin, F. Carlassare, M. T. Conconi, A. Capozzi, F. Majone, A. Guiotto, and F. Baccichetti, Photochem. Photobiol. 55 (1992), 221.

[17] F. Bordin, F. Dall'Acqua, and A. Guiotto, Pharmac Ther. 52 (1991), 331.

[18] F. Bordin, C. Marzano, F. Carlassare, P. Rodighiero, A. Guiotto, S. Caffieri, and F. Baccichetti, J Photochem. Photobiol., B:Biology 34 (1996), 159.

[19] M. A. Pathak and C. Carraro, The Biological role of Reactive Oxygen species in skin, p. 75, University of Tokyo Press, Tokyo, 1987, O. Hayaishi, S. Imamura and Y. Miyhachi (Eds.).

[20] B. Ortel and R. W. Gange, J. Invest. Dermatol. 94 (1990), 781.

[21] F. Dall'Acqua, D. Vedaldi, and S. H. Caffieri, The fundamental bases of phototherapy, p. 1, OEMF, Milan, 1996, G. Honisgmann, A. Jori, R. Young (Eds.).

[22] F. Bordin, F. Carlassare, L. Busulini, and F. Baccichetti, Photochem. Photobiol. 58 (1993), 133.

[23] S. Caffieri, personal communication.

[24] K. W. Kohn, Pharmac Ther. 49 (1991), 55.

[25] S. S. Sastry, H. P. Spielman, Q. S. Hoang, A. M. Philips, A. Sancar, and J. E. Hearst, Biochemistry 32 (1993), 5526. 


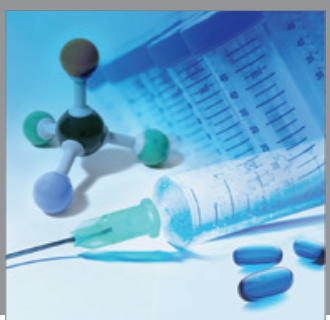

International Journal of

Medicinal Chemistry

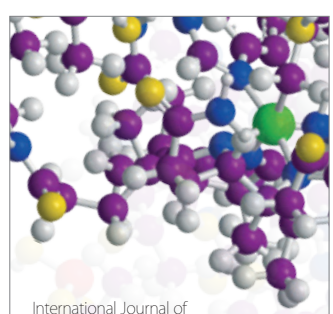

Carbohydrate Chemistry

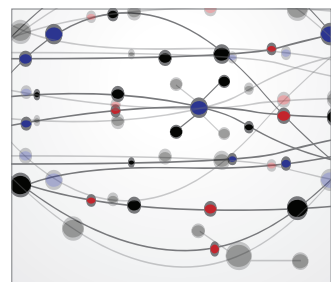

The Scientific World Journal
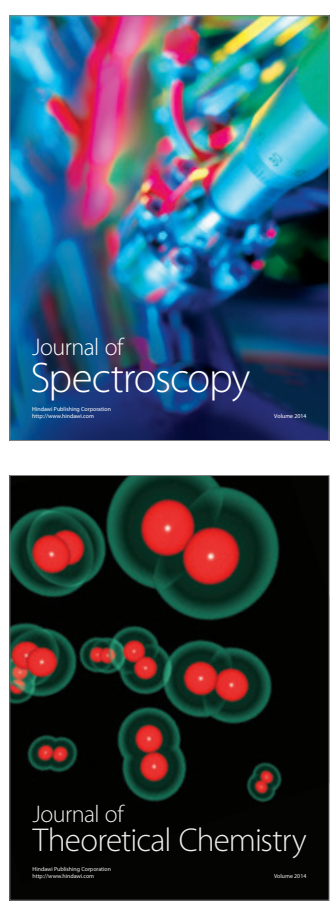
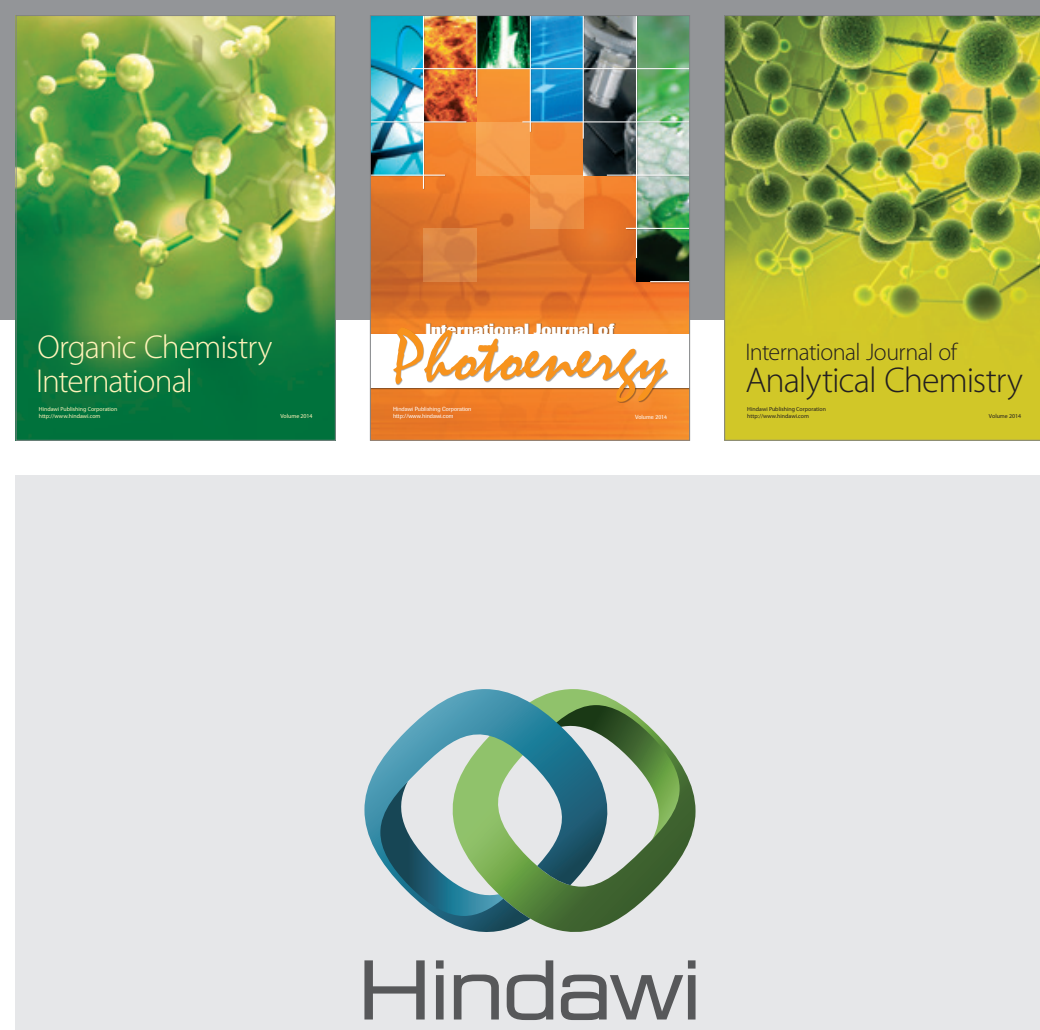

Submit your manuscripts at

http://www.hindawi.com
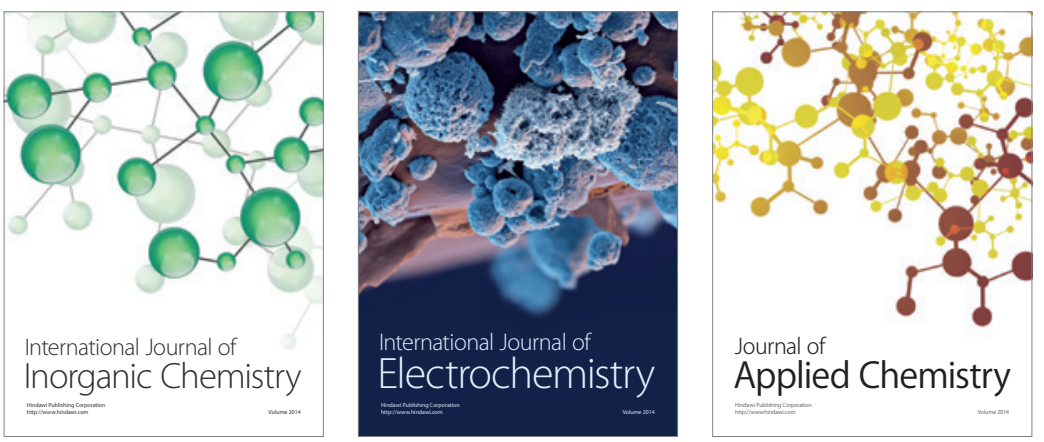

Journal of

Applied Chemistry
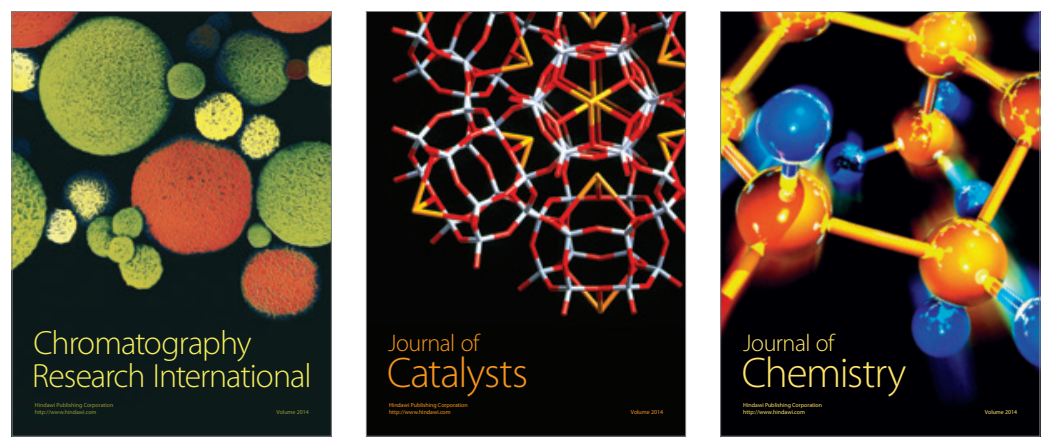
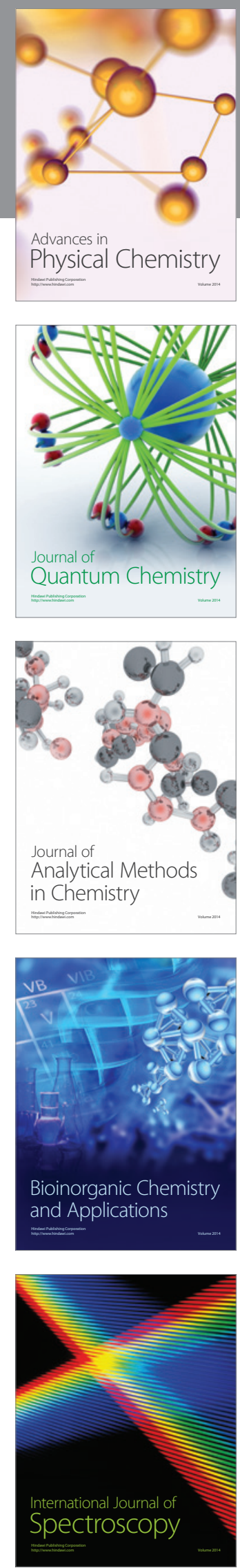